\title{
Virtual Synchronous Generator: A Control Strategy to Improve Dynamic Frequency Control in Autonomous Power Systems
}

\author{
Miguel Torres, Luiz A. C. Lopes \\ Department of Electrical and Computer Engineering, Concordia University, Montreal, Canada \\ Email:mi_torre@ece.concordia.ca, lalopes@ece.concordia.ca
}

Received February 28, 2013; revised March 28, 2013; accepted April 15, 2013

Copyright (C) 2013 Miguel Torres, Luiz A. C. Lopes. This is an open access article distributed under the Creative Commons Attribution License, which permits unrestricted use, distribution, and reproduction in any medium, provided the original work is properly cited.

\begin{abstract}
Ideally, diesel hybrid autonomous power systems would operate with high penetration of renewable energy sources such as wind and photovoltaic to minimize fuel consumption. However, since these are inherently intermittent and fluctuating, the grid-forming diesel engine generator sets are usually required to operate with larger amounts of spinning reserve, often at low loading conditions what tends to increases operating and maintenance costs. Frequency stability is of great concern in "small" systems, such as mini-grids, where any individual generator in-feed represents a substantial portion of the total demand. There, the initial rate of change of frequency is typically larger and a lower value of frequency can be reached in a shorter time than in conventional systems with all generation supplied by rotating machines, possibly resulting in under-frequency load shedding and tripping of renewable energy generators. The first part of this paper, discusses some general concepts regarding frequency stability in a diesel hybrid mini-grid and how energy storage systems can be used to enhance system performance. Then, a particular technique based on a virtual synchronous generator is presented and its effectiveness is demonstrated with simulation results.
\end{abstract}

Keywords: Frequency Control; Mini-Grid; Energy Storage; Inverter

\section{Introduction}

Historically, renewable energy sources (RESs) are integrated into autonomous power systems (mini-grids) already supplied by a diesel power plant with multiple diesel engine generator sets (gen-sets). With the addition of RES, it is more likely that the gen-sets will operate under low load conditions resulting in lower efficiency and potential of carbon build up and premature ageing of the diesel engines. Re-sizing of the gen-sets and implementation of appropriate cycling strategies, according to daily and seasonal variations of the mini-grid load and power output of the RESs to maintain optimum loading under various conditions, can help improve the RES contribution and displacement of diesel fuel [1]. Besides, one can also include short- or long-term energy storage systems (ESSs) and controllable (secondary) loads that can be used for improving stability and economic performance of the mini-grid. This can be done by a supervisory controller which should, at one level, (primary control) maintain grid stability and, at the other level, (secondary control) optimize the operation of the power sources, energy storage units and controllable loads, if applicable.

Figure 1 shows a schematic diagram of a supervisory controller of a diesel power plant. Each gen-set presents a primary controller, implemented in the governor, whose main function is to adjust the speed of the gen-set. Since most gen-sets employ a synchronous generator, the governor indirectly controls the frequency of the generated voltage. If the frequency is to be kept constant, an isochronous governor can be used. However, if multiple gen-sets are to operate in parallel, it is more convenient to use a droop based governor where the speed and frequency of a gen-set decrease as its output power increases. This behavior can be represented in steady-state by

$$
P=P_{\text {sch }}+\frac{1}{R}\left(f_{0}-f\right)
$$

where $P$ is the actual power supplied by the gen-set, $f$ is the grid frequency, $P_{\text {sch }}$ and $f_{0}$ are input values 


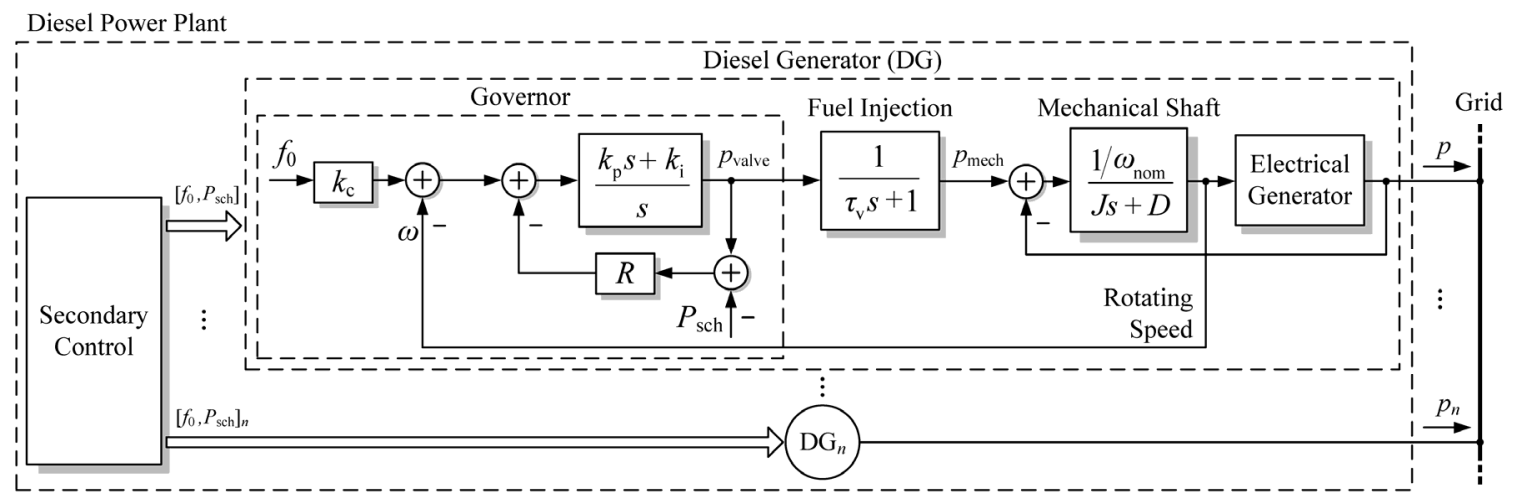

Figure 1. Diesel power plant.

that can be set by the secondary control and $R$ is the droop factor usually selected so that $f$ decreases by about $3 \%-5 \%$ as $P$ varies from no-load to full-load. In the isochronous mode, $R$ is set to 0 , meaning that the grid frequency will not change regardless of the output power level.

Frequency stability issues usually appear up to a few seconds following variations in the power demanded from the diesel plant. They are addressed primarily by the primary control blocks which should make sure that the rate of change of frequency (ROCOF) and frequency excursions remain within acceptable values. One important element in the variation of the grid frequency after variations in demand is the amount of kinetic energy stored in the rotating masses of the diesel power plant, which tend to reduce frequency variations. When a diesel hybrid mini-grid operates with high penetration of inertia-less power sources, such as photovoltaic (PV), frequency stability can deteriorate. Wind turbines contribute to a certain extent with inertial response, but not as a gen-set of equivalent power since they operate at low rotational speeds and many of the small ones are of the fixed pitch type, directly connected to the mini-grid via a squirrel-cage induction generator.

The secondary control, adjusts the values of and/or to bring the frequency of the mini-grid back to a desired value and/or to adjust the amount of power provided by each parallel gen-set so as to keep them loaded at safe and more economical levels as computed by an economic dispatch algorithm. However, due to a slow speed of response (bandwidth), the secondary control cannot make a meaningful contribution regarding frequency stability. One approach based on the use of a short-term ESS and that can reduce this problem is discussed in Section 3 .

Without the secondary control, the frequency of the mini-grid will vary according to the loading of the diesel power plant and the power vs. frequency droop characteristics of the gen-sets in operation. Although systems operating with tightly regulated frequencies are customary, some standards such as the European EN50160 only require the frequency in autonomous power systems to be within $\pm 2 \%$ of the rated value $(50 \mathrm{~Hz}$ ) during $95 \%$ of the week and within $15 \%$ during $100 \%$ of the week [2]. Besides, operation with variable frequency can be advantageous because it naturally conveys the information of shortage (low frequency) or excess (high frequency) of active power in the mini-grid. This would allow controllable loads, ESSs and power sources dispersed in the mini-grid to adjust their active power levels accordingly, without the need for a dedicated communication channel [3-5].

The supervisory control system of a diesel-hybrid mini-grid with short and long-term ESSs and controllable loads can be made very sophisticated, with multiple hierarchical control levels, employing modern information and communication technology (ICT) [6]. This paper however focuses on frequency transient stability issues which can be appropriately studied considering only the primary control only of the gen-sets and ESSs.

\section{Energy Storage Systems}

As mentioned before, the stochastic nature of wind and PV can put a lot of stress on the grid-forming diesel power plant to balance demand and supply. As a result, the system might present frequency stability (power quality) issues due to a lower amount of rotating masses (inertia), and require the diesel power plant to operate with higher spinning reserve (economical operation). The use of energy storage units can solve not only these problems but also minimize fuel consumption in the long term. However, since conventional battery storage is usually one of the most expensive components of a minigrid over its life-time, there are cost advantages to eliminating energy storage entirely, or incorporating only smaller units to assist with transient dynamics. Another way to avoid higher diesel based spinning reserve is load shedding, but its "cost" to system users, in terms of perceived loss of power quality and reliability, has to be balanced with the other options. 


\subsection{ESS Power Configurations}

The choice of the ESS configuration depends on the requirements of the application. Since in this particular case the objective is to improve the frequency stability of the diesel hybrid mini-grid and provide storage based spinning reserve, the discussion will be constrained to short-term storage media.

Short-term media like super-capacitors present fast dynamics, being able to supply and absorb large and frequent bursts of power, but present lower energy density than batteries [7]. Conversely, batteries present higher capacity and efficiency for storing energy which would be better employed in a more paced way. High speed flywheels could be a good choice for both requirements [8]. Another option would be to use hybrid ESS [7] like the ones shown in Figure 2, with two different types of storage technologies, batteries and super-capacitors (SC). The cascaded connection implies that the dc bus voltage, $v_{\mathrm{dc}}$, must be controlled by the converter $\mathrm{C} 1$, while $\mathrm{C} 2$ is controlled to supply the low frequency components of the current demanded by the dc-ac converter, lowering the stress on the batteries. A disadvantage is that converter $\mathrm{C} 1$ has to support all the current flow and, if it fails, then $\mathrm{C} 2$ cannot be used. On the other hand, in the parallel connection both converters can work independently and their state-of-charge (SoC) can be used to adjust their compensation efforts accordingly.

The dc-ac converter is controlled in the $P Q$ mode, injecting the required amounts of active $(P)$ and reactive $(Q)$ power independently. The references values for $P$ and $Q$ can be provided locally or sent by the supervisory controller of the mini-grid through a slow communication channel.

\subsection{ESS Control Strategies}

One of the most common strategies to generate the active power reference of distributed ESS ( $\left.p_{\text {ESSref }}\right)$ is frequency droop [9]. A major advantage of this technique is that allows the autonomous control of ESSs, i.e. no need for communication link, since the control relies on local measurements. However, in its simplest form (static frequency droop) it only guarantees frequency regulation support for a reduced amount of time, and it does not cope with the problem of dynamic frequency control. To address the latter, a more sophisticated frequency droop control is needed.

A strategy that indirectly deals with dynamic frequency control is the output power smoothing of intermittent sources [10]. This technique entails generating a suitable power reference for the ESS that cancels the high frequency components of the intermittent power. By doing this, the fast frequency variations induced by intermittent sources are attenuated. One disadvantage of this technique is that it requires the measuring of the output power of the intermittent source, which restricts the location of the ESS or establishes the need for a communication link to transmit the measurements.

Although different approaches to support dynamic frequency control can be found in the literature, one of the most discussed is the emulation of inertia [11]. In general, inertia emulation techniques support diesel generator response right after a load change occurs, keeping frequency under more restricted limits, and therefore contributing to maintain or enhance power quality. In the following section we discuss this technique as part of a control strategy called virtual synchronous generator.

\section{Virtual Synchronous Generator}

Virtual synchronous generator (VSG) is a control strategy that has been proposed to mitigate stability issues in power systems with large fraction of distributed generation [12]. The concept behind VSG is to control the grid-

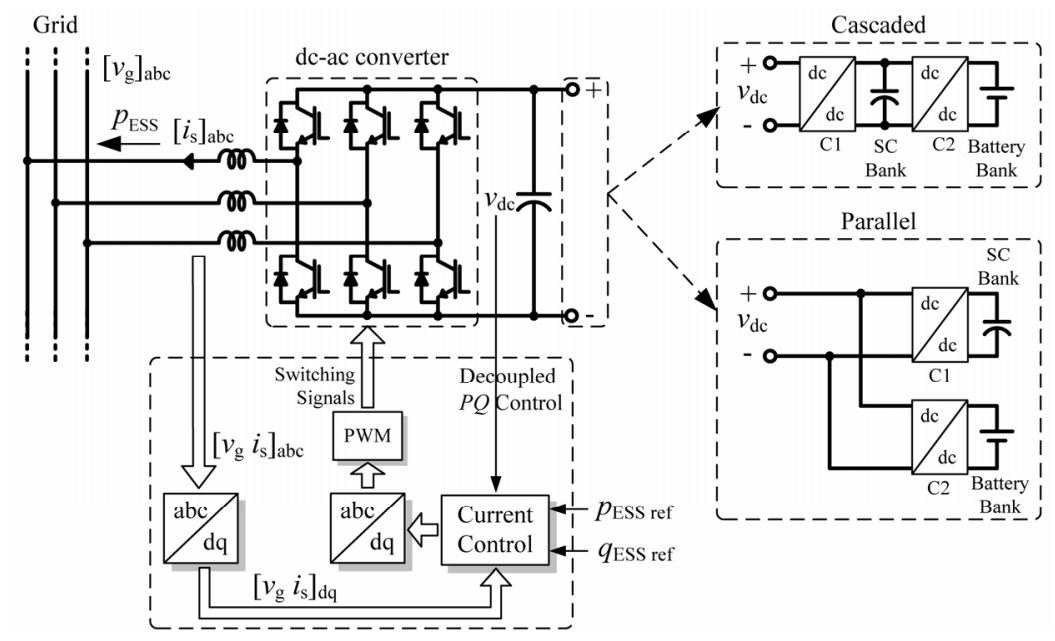

Figure 2. Hybrid ESS with two possible configurations and the $P Q$ control scheme for the dc-ac converter. 
interface converter of a distributed generator or ESS in order to emulate a synchronous generator or a desired characteristic of it. Different realizations of the VSG can be developed depending on the application. In this particular case, it is desirable to emulate characteristics that can be used to support frequency control, such as inertial response, damping and droop control. Therefore, the output power of the VSG can be synthesized according to:

$$
p_{\mathrm{VSG}}=\underbrace{\frac{f-f^{*}}{R}}_{\text {droop }} \underbrace{-k_{D}\left(f-f^{*}\right)}_{\text {damping }} \underbrace{-k_{J_{\mathrm{v}}} \frac{\mathrm{d} f}{\mathrm{~d} t}}_{\text {inertia }}
$$

where $f$ is the measured grid frequency. The droop term is calculated from a frequency droop curve as indicated in [9]. The other two terms in (2), damping and inertia, are present only during the transient, and therefore they are more related to the power capacity of the ESS rather than the energy capacity.

The damping term, with $k_{D}$ being the damping coefficient and $f^{*}$ the stabilization frequency, is the power that helps to attenuate oscillations. Note that in order to provide damping power in a proper way, the stabilization frequency should be known a priori. In the case of a system operating with isochronous primary frequency control, $f^{*}$ should be the nominal frequency of the system. However, in the case of droop control, the stabilization frequency will change with the load level and, therefore, an estimation technique is needed to determine its value.

Finally, the inertial term, with $k_{J v}$ representing the virtual inertia, emulates the inertial response of a conventional generator, whose main effects over the system frequency are: decreasing the rate of change and the maximum deviation following a power disturbance. In order to limit the extension of the paper, only the effects of the emulation of inertia on the dynamic frequency control are presented. To the best knowledge of the authors, studies published so far have focused on techniques that consider a constant virtual inertia. The strategy proposed in this paper entails the online modification of this parameter.

\subsection{Self-Tuning Virtual Inertia}

The idea was motivated by the fact that when frequency starts to deviate from steady-state, a larger inertia would present a stronger opposition to the speed change of generators, limiting the initial rate of change of frequency and its peak deviation. On the other hand, when frequency starts to return to steady-state after the peak deviation, a larger inertia is no longer required. Figure 3 depicts a block diagram of the VSG control for emulating inertia. The grid frequency is locally measured at the point where the ESS is connected and then, based on the

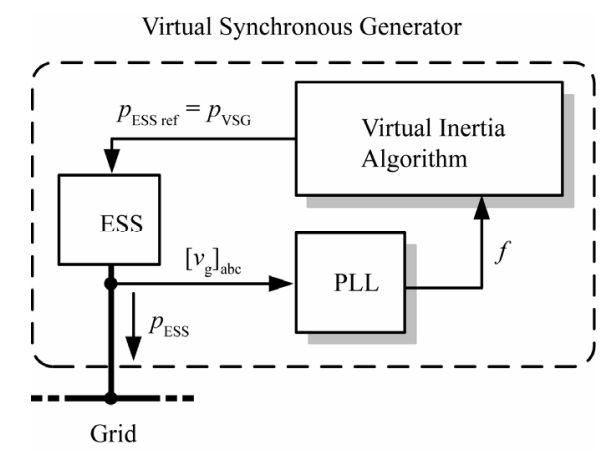

Figure 3. Block diagram of a VSG control performing only the virtual inertia function.

measured frequency, the virtual inertia algorithm calculates the output power of the VSG, which turns out to be the reference for the output power of the ESS.

\subsubsection{Linear Variation}

This algorithm starts to increase the value of the virtual inertia if the frequency varies faster than a predefined maximum rate (MAX). On the other hand, it starts to linearly decrease the virtual inertia when the frequency varies slower than a minimum rate (MIN). The algorithm is executed in every sampling time of the controller and it can be summarized in the following expressions:

$$
\begin{array}{r}
\left|\frac{\mathrm{d} f}{\mathrm{~d} t}\right| \begin{cases}>\text { MAX } & i=i+1 \\
<\text { MIN } & i=i-1\end{cases} \\
k_{J_{\mathrm{v}}}=\boldsymbol{k}(i) \\
p_{\mathrm{VSG}}=k_{J_{\mathrm{v}}} \pi \frac{\mathrm{d} f}{\mathrm{~d} t}
\end{array}
$$

where $\boldsymbol{k}$ is a predefined monotonically increasing vector of values of virtual inertia, $i$ is an integer counter used in the algorithm to access the elements of the vector $\boldsymbol{k}$, and $\pi$ is the conversion factor between frequency and generator speed.

\subsubsection{Optimal Inertia}

This algorithm finds a value of virtual inertia, from a predefined finite set of values, which minimizes the cost function $g$. The optimization problem is solved in every sampling time and it can be formulated as follows:

$$
\begin{gathered}
\min _{\substack{k_{J_{\mathrm{v}}} \in \mathbf{k} \\
\alpha>0}} g=\alpha \pi\left|\frac{\mathrm{d} f}{\mathrm{~d} t}\left(+T_{s}, k_{J_{\mathrm{v}}}\right)\right|+\left|k_{J_{\mathrm{v}}}\right| \\
p_{\mathrm{VSG}}=k_{J_{\mathrm{v}}} \pi \frac{\mathrm{d} f}{\mathrm{~d} t}
\end{gathered}
$$

where $\mathrm{d} f / \mathrm{d} t\left(+T_{s}, k_{J_{\mathrm{v}}}\right)$ is the one sampling time ahead prediction of the rate of change of frequency. This prediction is based on Equation (2.1) in [13] that describes the dominant dynamics of the system frequency. 
On the other hand, the second term of the cost function is included to represent the output power and the weight factor $\alpha$ determines the relative importance between the two terms.

\section{Performance Verification}

To verify the performance enhancement offered by the proposed control strategy, a simulation is conducted for the system of Figure 4, which represents a diesel-hybrid autonomous power system. It consists in two identical diesel gen-sets as primary sources of energy (DG1, DG2), a type 1 wind generator (WG) used to complement power production, an energy storage system, and several resistive loads. The main parameters of the diesel gensets and wind generator are presented in Tables 1 and 2 respectively. Since the secondary control is slower than the primary one, it will have no impact over the system frequency during the time frame of the simulations and it will not be considered in this study. The system is simulated for the critical case when only one gen-set is in operation (DG1).

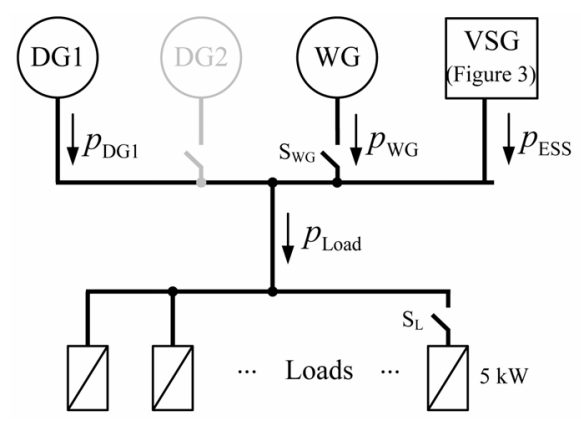

Figure 4. Schematic diagram of the diesel-hybrid autonomous power system.

Table 1. Parameters of diesel generators.

\begin{tabular}{lccc}
\hline Description & Symbol & Value & Unit \\
\hline Rated power & n.a. & 37.7 & $\mathrm{~kW}$ \\
Nominal speed & n.a. & 1800 & $\mathrm{rpm}$ \\
Nominal frequency & n.a. & 60 & $\mathrm{~Hz}$ \\
Inertia & $J$ & 1.6 & $\mathrm{kgm}^{2}$ \\
Friction losses & $D$ & 0.18 & $\mathrm{kgm}^{2} / \mathrm{s}$ \\
Scheduled power & $P_{\mathrm{sch}}$ & 0 & $\mathrm{~kW}$ \\
No-load frequency & $f_{0}$ & 63 & $\mathrm{~Hz}$ \\
Droop coefficient & $R$ & 0.5 & $(\mathrm{kWs})^{-1}$ \\
Conversion factor & $k_{\mathrm{c}}$ & $\pi$ & $\mathrm{n} . \mathrm{a}$. \\
Proportional gain & $k_{\mathrm{p}}$ & 1.9 & $\mathrm{kWs}$ \\
Integral gain & $k_{\mathrm{i}}$ & 5.7 & $\mathrm{~kW}$ \\
Time constant & $\tau_{\mathrm{v}}$ & 43 & $\mathrm{~ms}$ \\
\hline
\end{tabular}

Table 2. Parameters of wind generator.

\begin{tabular}{ccc}
\hline Description & Value & Unit \\
\hline Power at $9.5 \mathrm{~m} / \mathrm{s}$ & 50 & $\mathrm{~kW}$ \\
Cut-in wind speed & 3.5 & $\mathrm{~m} / \mathrm{s}$ \\
Nominal frequency & 60 & $\mathrm{~Hz}$ \\
Power regulation & Stall control & $\mathrm{n} . \mathrm{a}$. \\
Rotor speed & 42 & $\mathrm{rpm}$ \\
Rotor diameter & 19 & $\mathrm{~m}$ \\
Equivalent Inertia & 5.83 & $\mathrm{kgm}^{2}$ \\
\hline
\end{tabular}

The proposed algorithms are compared with the constant virtual inertia in two cases of transient condition: a load increase and wind variations. In all cases, the constant virtual inertia has a fixed value of $5 \mathrm{kgm}^{2}$, the vector $\boldsymbol{k}$ is defined with 20 elements linearly spaced from 0 to $5 \mathrm{kgm}^{2}$, the sampling time is $T_{s}=10 \mathrm{~ms}, \mathrm{MIN}=0.1$ $\mathrm{Hz} / \mathrm{s}, \mathrm{MAX}=1 \mathrm{~Hz} / \mathrm{s}$, and $\alpha=4$.

\subsection{Load Increase}

The system starts operating with DG1 supplying a load of $25 \mathrm{~kW}$ and with $p_{\mathrm{ESS}}=0 \mathrm{~kW}$. The switch SL is closed at $t=0 \mathrm{~s}$, producing a load increase of $5 \mathrm{~kW}$. Figure 5 shows the simulation results.

In comparison to the gen-set only response (waveform labeled DG1), all the inertia emulation methods reduce the maximum frequency deviation. The rate of change of frequency is also reduced. However the constant and variable inertia result in noticeable oscillations during the first $0.2 \mathrm{~s}$. This might be a consequence of the numerical calculation of the derivative with insufficient prefiltering of the measured frequency. On the other hand, the optimal algorithm did not present much oscillation, which might be because it used a first order model to calculate the derivative of the frequency and its prediction.

In the bottom plot it is observed that the optimal inertia algorithm started to decrease the value of inertia $(\mathrm{t}$ $\approx 0.4 \mathrm{~s})$ before than the variable inertia algorithm $(\mathrm{t} \approx 2.1$ s). The ESS output power started to decrease accordingly, and therefore the frequency deviation was slightly greater for the optimal inertia.

\subsection{Wind Variations}

The system starts operating with DG1 and the WG supplying a load of $30 \mathrm{~kW}$. The ESS is enabled at $t=0.1 \mathrm{~s}$. The wind speed varies according to a real wind profile obtained from [14]. Figure 6 shows the simulation results. It is observed that all the algorithms of inertia emulation achieved a similar performance in terms reduction of frequency variations. However, from the middle plot, it can be noted that the optimal inertia 

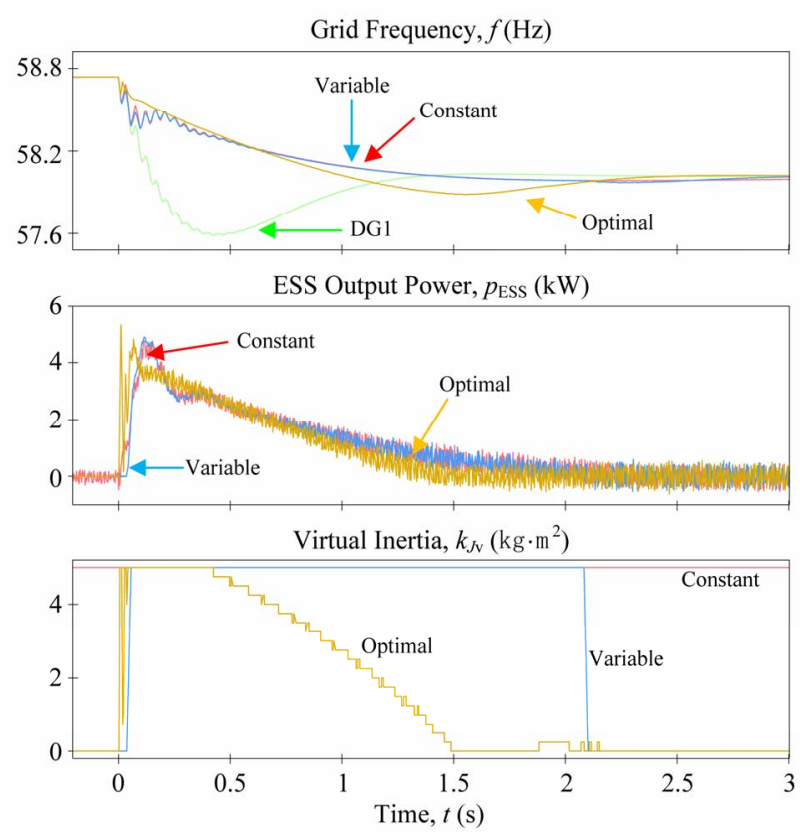

Figure 5. Comparison of virtual inertia strategies for a load increase.
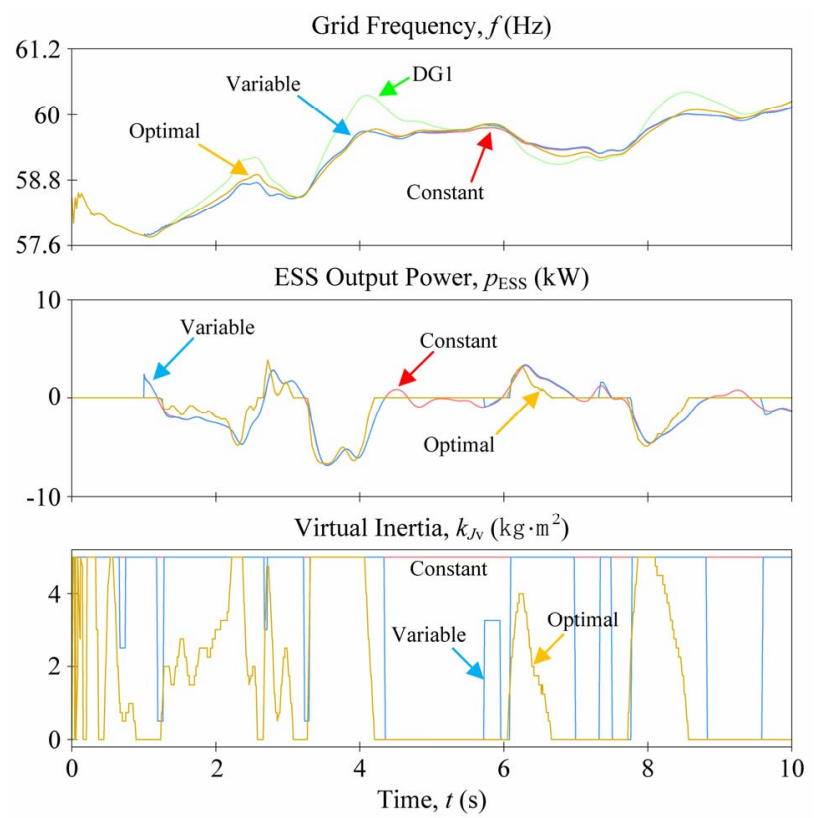

Figure 6. Comparison of virtual inertia strategies for wind variations.

algorithm used less power.

\section{Conclusion}

This paper addressed the issue of dynamic frequency control in diesel-hybrid autonomous power systems (mini-grids) with intermittent renewable energy sources (RES). It discussed basic concepts related to the configuration and control of a diesel power plant and energy storage systems (ESSs) that can enhance system stability and reduce fuel consumption. While controlling a ESS so as to smooth the fluctuating power of a co-located RES is beneficial, it would not mitigate problems caused by load variations, that are relatively large and common in remote community type systems. The control strategy called virtual synchronous generator (VSG), which can tackle both problems was described. Then, a case study of a wind-diesel system with a short-term ESS controlled as a virtual inertia, a sub-set of a VSG, was presented. Simulation results showed that an ESS controlled as a virtual inertia can enhance the dynamic frequency control of a mini-grid subject to fluctuating renewable energy and load variations. The concept of variable/optimal inertia was introduced in this paper. In general, it provided an improvement in the grid frequency response while requiring less power as compared to the conventional fixed virtual inertia scheme.

\section{REFERENCES}

[1] F. Katiraei and C. Abbey, "Diesel Plant Sizing and Performance Analysis of a Remote Wind-Diesel Microgrid," IEEE Power Engineering Society General Meeting, Tampa, 24-27 June 2007, pp. 1-8.

[2] European Committee for Electrotechnical Standardization, "EN50160 European Standard on Voltage Characteristics of Electricity Supplied by Public Distribution Systems," European Committee for Electrotechnical Standardization, Brussels, 2010.

[3] R. Tonkoski and L. A. C. Lopes, "Enhanced Part Load Operation of Hybrid Mini-Grids with High Penetration of Photovoltaics," 3rd Brazilian Conference on Solar Energy, Belém, 21-24 September 2010.

[4] K. Elamari and L. A. C. Lopes, "Frequency Based Control of Electric Water Heaters in Small PV-Diesel Hybrid Mini-Grids," 25th Canadian Conference on Electrical and Computer Engineering, Montréal, 29 April-2 May 2012.

[5] L. A. C. Lopes and M. Dalal-Bachi, "Economic Dispatch and Demand Side Management via Frequency Control in PV-Diesel Hybrid Mini-Grids," 6th European Conference on PV-Hybrid and Mini-Grids, Chambéry, 24-26 April 2012.

[6] F. Katiraei, R. Iravani, N. Hatziargyriou and A. Dimeas, "Microgrids Management," IEEE Power and Energy Magazine, Vol. 6, No. 3, 2008, pp. 54-65. doi:10.1109/MPE.2008.918702

[7] S. Lukic, S. Wirasingha, F. Rodriguez, J. Cao and A. Emadi, "Power Management of an Ultracapacitor/Battery Hybrid Energy Storage System in an HEV," IEEE Vehicle Power and Propulsion Conference (VPPC'06), Windsor, 6-8 September 2006.

[8] N. Hamsic, A. Schmelter, A. Mohd, E. Ortjohann, E. Schultze, A. Tuckey and J. Zimmermann, "Increasing Renewable Energy Penetration in Isolated Grids Using a Flywheel Energy Storage System," International Confer- 
ence on Power Engineering, Energy and Electrical Drives, 12-14 April 2007, pp. 195-200.

[9] P. C. Loh and F. Blaabjerg, "Autonomous Control of Distributed Storages in Microgrids," 2011 IEEE 8th International Conference on Power Electronics and ECCE Asia (ICPE ECCE), Jeju, 30 May- 3 June 2011, pp. 536-542.

[10] W. Li, G. Joós and C. Abbey, "Wind Power Impact on System Frequency Deviation and an ESS Based Power Filtering Algorithm Solution," 2006 IEEE Power Systems Conference and Exposition (PSCE'06), Atlanta, 29 October-1 November 2006, pp. 2077-2084.

[11] Y. Z. Sun, Z. S. Zhang, G. J. Li and J. Lin, "Review on Frequency Control of Power Systems with Wind Power
Penetration," 2010 International Conference on Power System Technology (POWERCON), Hangzhou, 24-28 October 2010.

[12] Q. C. Zhong and G. Weiss, "Synchronverters: Inverters That Mimic Synchronous Generators," IEEE Transactions on Industrial Electronics, Vol. 58, No. 4, 2011, pp. 1259-1267. doi:10.1109/TIE.2010.2048839

[13] H. Brevani, "Robust Power System Frequency Control," Springer Science+Business Media, New York, 2009.

[14] "Renewable Energy Cheaper than Coal," Wind Data Files. http://code.google.com/p/google-rec-csp/downloads/list 